\title{
Optical and Thermal Characteristics of Porous Anodic Aluminum Oxide for Photothermal Applications
}

\author{
Trong-Linh Nguyen, ${ }^{1,2}$ Chu-Chiang Chou, ${ }^{1}$ Te-Hua Fang, ${ }^{1}$ and Tsung-Chieh Cheng ${ }^{1 *}$ \\ ${ }^{1}$ Department of Mechanical Engineering, National Kaohsiung University of Science and Technology, \\ No. 415, Jiangong Rd., Sanmin Dist., Kaohsiung City 807, Taiwan \\ ${ }^{2}$ Faculty of Mechanical Engineering, Hung Yen University of Technology and Education, \\ Khoai Chau District, Hung Yen Province 17817, Vietnam
}

(Received April 3, 2021; accepted November 16, 2021)

Keywords: anodic oxidation, aluminum alloy, solar thermal absorber

Using aluminum alloy 6061-T6 as the substrate and sulfuric acid $(0.3 \mathrm{M})$ anodizing technology, we formed nanoporous anodic aluminum oxide (NPAAO) films for solar absorption and photothermal sensor applications in a medium temperature range. We found that the diameter and thickness of aluminum oxide increased with increasing applied anodic voltage. The increase in the applied anodic voltage, which increased the NPAAO film thickness, enhanced absorption over the solar spectral range. Therefore, the solar absorptivity increased with the thickness of the NPAAO film, and more of the penetrating light was trapped by the thicker NPAAO film due to its greater depth and higher pore density. Moreover, the transient temperature of the NPAAO films was measured under $500 \mathrm{~W}$ halogen light illumination to investigate the photothermal efficiency of the films. The results indicated that the steady-state temperature increased with the absorptivity of the NPAAO films. The mean reflectance of the aluminum alloy specimens in the spectral range of $250-850 \mathrm{~nm}$ was greater than $72 \%$ before the anodizing oxidation process and less than $7.7 \%$ after the process. Therefore, the applied anodic voltage, which changes the pore density and the thickness of the anodized film, is an important factor determining the solar absorption performance of NPAAO films.

\section{Introduction}

Solar absorption coating technology has been of great interest in recent years because it has been widely used in green energy, optical industries, and sensor applications, such as photothermal sensors, IR sensors, pyranometers, and solar cells. Photothermal sensors are designed to efficiently detect light intensity changes that are not easily noticeable and convert light energy into heat energy. The solar-selective absorbing coating films should have efficient solar photothermal conversion and thus must have high solar absorptivity and low thermal emissivity, especially at high temperatures. ${ }^{(1,2)}$ A spectrally selective surface should capture the maximum solar energy in the high-intensity visible and near-IR spectral regions. Minimizing the primary reflection loss at the front surface of solar thermal layers has always been a key challenge in improving power conversion efficiency. One way to increase the efficiency is to *Corresponding author: e-mail: tcchengme@nkust.edu.tw https://doi.org/10.18494/SAM3545 
reduce optical loss and achieve effective light trapping, such as by using antireflection (AR) coatings. ${ }^{(3)}$ Several AR methods have been reported to reduce surface reflection loss, such as the deposition of a single-layer or multilayer AR coating. Two different approaches are available for fabricating AR coatings. One is coating with multilayer films and the other is to form a subwavelength AR nanostructure on the surface of devices. ${ }^{(4)}$ When light crosses a boundary from one medium to another, some light is reflected and some light is transmitted. Theoretically, optical reflection can be efficiently suppressed if the refractive index of the coating is equal to the geometric mean of the refractive indices of the two media at the interface and the thickness of the coating is a quarter of the wavelength of light. ${ }^{(5)}$ Introducing a gradual change in the refractive index with an AR layer can suppress reflection. ${ }^{(6-8)}$ Therefore, a multilayer optical coating usually consists of a stack of several layers of absorbing materials with different refractive indices. Moreover, alternate layers of dielectrics and semi-transparent metals can be used as interference stacks to maximize absorption. The dielectrics are selected to strongly absorb visible light, and the metals are selected to reflect IR light. AR coatings are usually formed on the surface of such stacks to improve the transmission to the absorbing layers. ${ }^{(9)}$

Smooth surfaces reflect more light than rough ones, so an increase in roughness is necessary to reduce the reflection of surfaces. As a result, the second approach is to form micro/ nanostructures on the surface of materials to fabricate AR layers. Nature routinely produces nanostructured surfaces, so-called "moth-eye structures", which can reduce reflection by gradually changing the effective refractive index between a dielectric medium and air; thus, it is easy to fabricate such AR layers. Moreover, to reduce the reflectance of materials and increase the power generation efficiency of silicon-based solar cells, sub-wavelength structures (SWSs) that are smaller than the wavelength of light and have a periodic arrangement have been produced on the surface of materials to generate a strong AR effect. ${ }^{(10)}$ These SWSs can simultaneously and dramatically suppress the reflection and increase the light transmission at the interface over a wide range of angles and wavelengths because the surface has many tunable factors, such as period, depth, and cross-sectional geometry. ${ }^{(11)}$ These textured surfaces trap light, leading to broadband suppression of the reflection. Therefore, an AR layer can be realized by forming nanostructures, sub-wavelength pitches, gratings, or protrusions with heights of wavelength order. ${ }^{(12)}$ Furthermore, sub-wavelength tapered nanostructures have been integrated on a grating surface to provide adiabatic impedance matching in the form of a gradient-index medium, and the fabricated nanostructured grating suppressed reflection by two orders of magnitude over a broad range of wavelengths and incident angles. ${ }^{(13)}$

There are several techniques for fabricating periodic nanoscale patterns, such as photolithography, ${ }^{(14-16)}$ electron beam lithography, ${ }^{(17,18)}$ use of a focused ion beam, ${ }^{(19,20)}$ microcontact printing, ${ }^{(21,22)}$ inductively coupled plasma-reactive ion etching (ICP-RIE), ${ }^{(23)}$ and electron cyclotron resonance (ECR) plasma etching. (24) Although these methods can be used to fabricate nanoscale patterns precisely, it is not easy to fabricate patterns on a large-area structure because the process is time-consuming and very expensive. Therefore, in this study, we used a low-cost and simple anodizing process to fabricate a nanoporous surface on aluminum as an AR layer that could achieve a high-efficiency photothermal material for sensor applications. This method can also easily form an AR layer on any curved surface. 


\section{Materials and Methods}

\subsection{Experiments}

In this work, a set of optically lossy porous alumina [i.e., anodic aluminum oxide (AAO)] films were fabricated on top of aluminum substrates. To obtain a uniform distribution of the electric field on the surface during the anodizing process, a $3 \times 3 \mathrm{~cm}^{2}$ commercial 6061-T6 aluminum alloy was polished to form a flatter and smoother surface before anodizing. First, No. 600 sandpaper was used to polish the aluminum alloy, then $0.3 \mu \mathrm{m}$ aluminum oxide powder was used for fine polishing. After the polishing, sodium hydroxide, acetone, and nitric acid were used to wash and clean the specimen. The specimen was dipped into an electrolyte of perchloric acid and alcohol as the anode and then electrified at $10 \mathrm{~V}$ for $5 \mathrm{~min}$ for electrolytic polishing to obtain a smooth surface. Then, the specimen was placed at the anode and subjected to the anodizing process, where the cathode was a platinum sheet. Sulfuric oxide $(0.3 \mathrm{M})$ was used as the anodic electrolyte and the DC bias was fixed at 40, 50, or $60 \mathrm{~V}$. After the anodizing process, the specimen was removed and washed with acetone, and ultrasonic waves were also applied to remove the adsorbed pollutants.

\subsection{Measurement systems}

The surface microstructure of the specimens was observed by field-emission scanning electron microscopy (SEM) (JEOL, JSM-6700F, Japan). The spectral reflectance (R) of the specimens was recorded by a UV-VIS-NIR spectrophotometer (Shimadzu, UV-2450, Japan) with an integrating sphere detector. In addition, the anodic specimen was exposed to a $500 \mathrm{~W}$ halogen lamp to simulate stable solar light, and the distance between the halogen lamp and the solar cell was fixed at $0.5 \mathrm{~m}$. To estimate the photothermal conversion efficiency, a data logger (Yokogawa MV100) with a voltage sensitivity of $0.01 \mathrm{mV}$ and a current sensitivity of $0.01 \mathrm{~A}$ was used to record voltage and current data. The transient temperature was measured using K-type thermocouples connected to a temperature logger with $0.1 \mathrm{~K}$ accuracy. ${ }^{(5)}$ The data were then transferred to a PC to record the transient temperature profile of the nanoporous anodic aluminum oxide (NPAAO) films as a reference to evaluate photothermal conversion efficiency.

\section{Results and Discussion}

Figure 1 shows SEM images of anodized 6061-T6 aluminum alloy with and without polishing. The roughness of the unpolished specimen is higher than that of the polished specimen after the anodizing process. The surface of the unpolished aluminum alloy specimen in Fig. 1(a) has many bumpy defects, and there is no uniform structure on the surface. The local accumulation of alloying elements in a given metal/oxide interface region influences local oxidation rates and modifies the oxide morphology. Different oxidation rates of the alloy matrix and regions with impurities can result in differences in the morphology of the anodized film. ${ }^{(25)}$ Moreover, during the anodizing process, the electric field is concentrated on the scratch marks and defects of the 


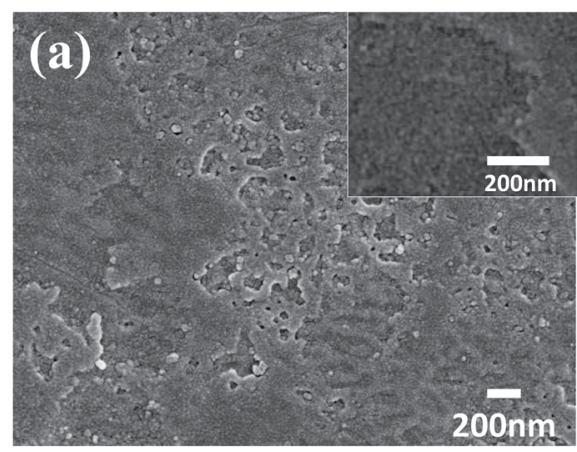

(a)

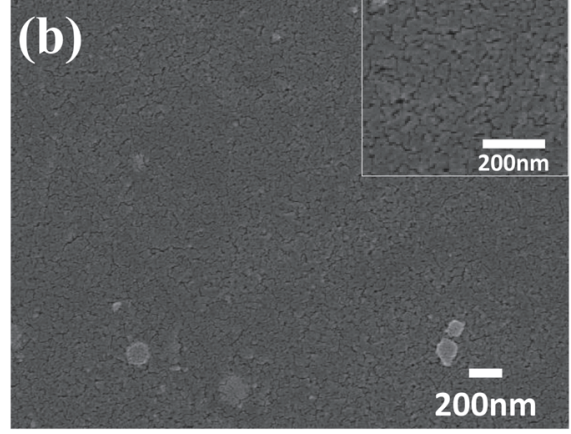

(b)

Fig. 1. SEM images after anodizing of aluminum alloy (a) without and (b) with polishing.

surface, which can easily form pores on the hollows of the surface. This phenomenon causes an uncontrollable pore distribution and arrangement of the oxide layer. To avoid the effect of defects and grow controllable pores on the aluminum alloy surface, polishing is necessary to reduce these scratches and defects. This process produces uniform pores after the anodizing process, as shown in Fig. 1(b). However, unlike conventional AAO membranes having hexagonal periodicity, the synthesized anodic aluminum alloy film exhibits irregularly distributed nanopores with different sizes.

Figure 2 shows the measured spectral reflectance and transient temperatures of NPAAO films under $500 \mathrm{~W}$ halogen lamp irradiance. The UV-VIS-NIR spectrophotometer (integrating sphere detector) was used here to rigorously characterize the light absorption of the specimens in a wide wavelength range of 250-2500 nm, as shown in Fig. 2(a). The mean reflectance of the untreated aluminum alloy was $63.5 \%$, and after the polishing, the mean reflectance increased to $72 \%$ because of the reduced scattering of the flat surface. When the anodizing process was performed to generate the porous surface of the aluminum alloy, the mean reflectance of the unpolished and polished specimens was reduced to 11.8 and $8.8 \%$, respectively. After the anodizing process, the polished specimen showed the lowest reflectance and the greatest surface absorption. Fig. 2(b) presents the transient temperature variation for different conditions of the aluminum alloy treatment. Interestingly, the steady-state temperature difference between the untreated sample and the polished and anodized sample exceeded $230{ }^{\circ} \mathrm{C}$. These results also indicate that the photothermal conversion efficiency is proportional to the absorption of the aluminum alloy. The polished and anodized sample showed the highest steady-state temperature, which may be attributed to the nanopores being grown on the surface of the aluminum alloy. These nanopores can change the effective refractive index of the anodized film so that it matches the refractive indices of the substrate and air, leading to a significant decrease in reflectivity, thus improving light trapping and photothermal efficiency. ${ }^{(26)}$

Figure 3 shows SEM images of the NPAAO film at different applied anodic voltages. The growth mechanism of the NPAAO film depends on the equilibrium between the electrical fieldoxide formation at the metal/oxide interface and the oxide dissolution at the electrolyte/oxide interface. The pore size, interpore distance, pore thickness, and electrolyte temperature 


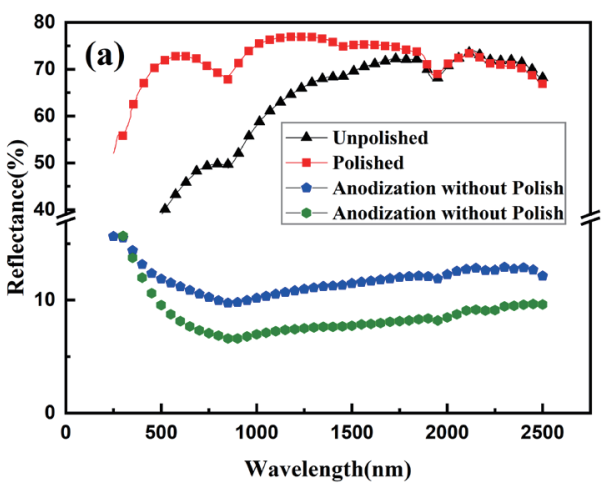

(a)

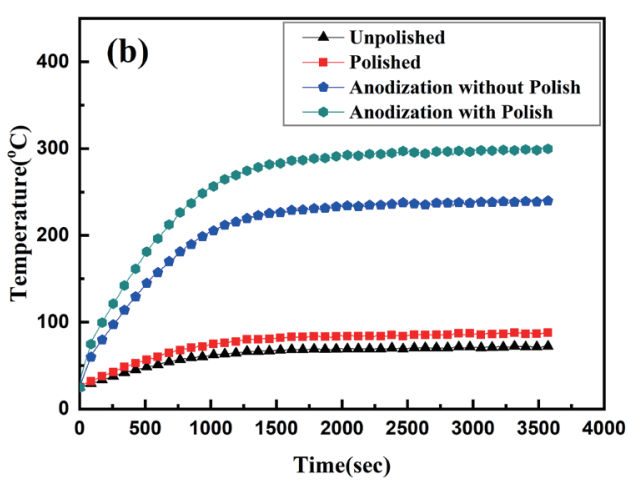

(b)

Fig. 2. (Color online) (a) Spectral reflectance and (b) transient temperature profiles of NPAAO films.

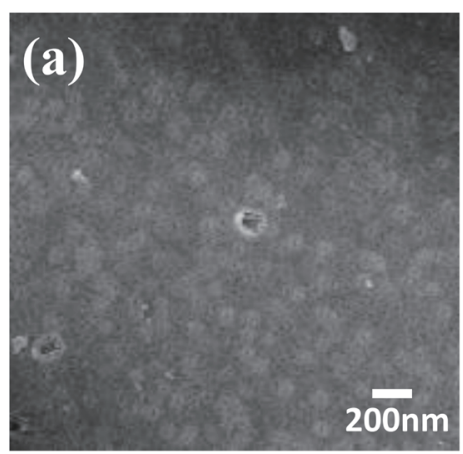

(a)

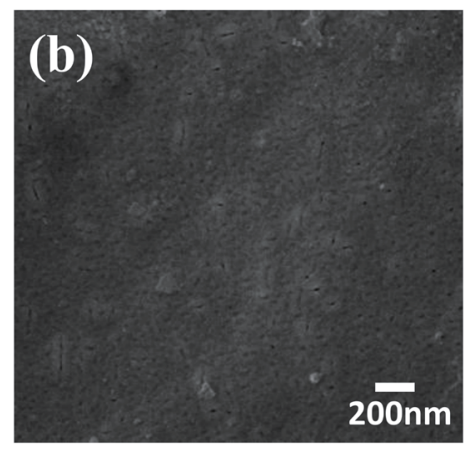

(b)

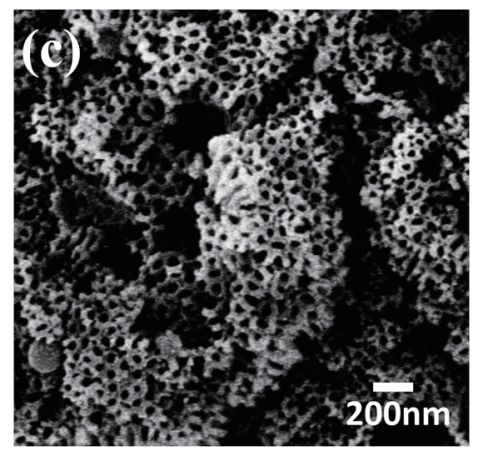

(c)

Fig. 3. SEM images of anodic NPAAO films with applied anodic voltages of (a) $40 \mathrm{~V}$, (b) $50 \mathrm{~V}$, and (c) $60 \mathrm{~V}$.

increased with the applied anodic voltage. As shown in Figs. 3(a) and 3(b), the average pore size was about 32 and $48 \mathrm{~nm}$ at the applied anodic voltages of 40 and $50 \mathrm{~V}$, respectively. When the applied anodic voltage was increased to $60 \mathrm{~V}$, the pore size was about $85 \mathrm{~nm}$, but this large voltage also induced the dissolution of the NPAAO film and the pore structure began to collapse, as shown in Fig. 3(c).

Figure 4 shows the measured spectral reflectance and transient temperature of the NPAAO films with different applied anodic voltages. When light penetrates the anodic film, the effective depth of the periodic structure exceeds the wavelength of the incident light due to the pore density and thickness of the anodic film, thereby increasing the probability that incident light is absorbed. Fig. 4(a) indicates that the mean AR characteristic of the specimens became more robust when the applied anodic voltage was increased to $50 \mathrm{~V}$. The mean reflectance was $72 \%$ for the polished surface without anodizing and 7.85 and $7.77 \%$ for the surfaces anodized at applied anodic voltages of 40 and $50 \mathrm{~V}$, respectively. As the applied anodic voltage increased, greater effective depths of the periodic structure with deeper holes were obtained, thereby increasing the probability that incident light is absorbed. With increasing depth of the periodic 


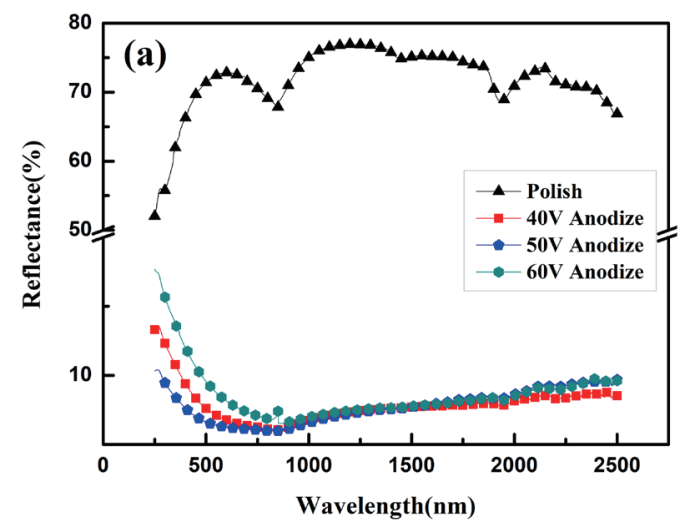

(a)

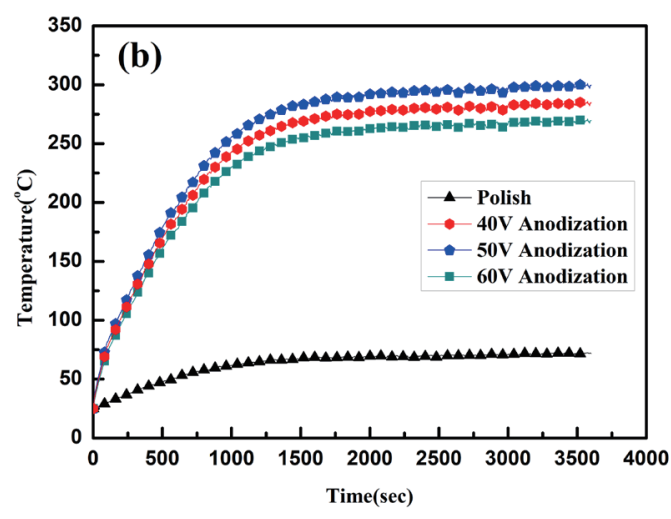

(b)

Fig. 4. (Color online) (a) Spectral reflectance and (b) transient temperature profiles of NPAAO films with different applied anodic voltages.

structure, the reflectance decreased then approached a stable value. ${ }^{(27)}$ These results show that anodizing can significantly increase the absorption of aluminum alloy and that absorption increases with increasing applied anodic voltage. Moreover, residual impurities and alloying elements in the 6061-T6 aluminum alloy will increase the material loss, which depends on the volume fraction of air and lossy alumina, ${ }^{(5)}$ and also increase the absorbance of the anodic aluminum alloy. However, the reflectance of the NPAAO film increased when the applied anodic voltage was increased to $60 \mathrm{~V}$, as shown in Fig. 4(a). Owing to the high electrostatic biasing field in the anodizing process, the active surface oxidation results in rapid Joule heating and causes morphological damage to the surface, as shown in Fig. 3(c). This phenomenon also decreased the steady-state temperature of the NPAAO film. Furthermore, the transient temperature profiles in Fig. 4(b) also indicate that the steady-state temperature is proportional to the absorption of the NPAAO surface, which is related to photothermal efficiency. The steadystate temperature increased dramatically after anodizing and the maximum temperature of these anodic aluminum alloy specimens reached $290^{\circ} \mathrm{C}$. In other words, the photothermal efficiency of aluminum alloy can be easily increased by this low-cost and simple anodizing process.

\section{Conclusions}

Antireflective NPAAO films on 6061-T6 aluminum alloy were investigated in this study. Sulfuric acid $(0.3 \mathrm{M})$ was used as an electrolyte for the anodizing process to generate NPAAO on the surface. It was found that polishing is important for producing a uniform nanopore structure on the aluminum alloy. Without polishing, it is not easy to form a uniform nanoporous structure because of the roughness and defects of the surface. Moreover, increasing the applied anodic voltage increased the thickness of the nanopores and decreased the reflectance of the NPAAO film because of the increased probability of incident light absorption and material loss, which depends on the volume fractions of air and lossy alumina. Consequently, the photothermal efficiency also increased with increasing applied anodic voltage when the applied anodic voltage 
was less than $60 \mathrm{~V}$. The maximum steady-state temperature of the anodized specimens reached about $290{ }^{\circ} \mathrm{C}$. As a result, anodizing of the surface of lossy 6061-T6 aluminum may provide an effective platform to tune the absorption and reflection properties of NPAAO surface coatings, improving their sensitivity. Therefore, the anodizing process is a quick and low-cost way to fabricate AR layers on aluminum alloys for solar absorption and photothermal sensor applications.

\section{Acknowledgments}

This work was supported in part by the Ministry of Science and Technology of the Republic of China, Taiwan, ROC, under Contract No. MOST 108-2221-E-992-061-MY2. The authors also thank the Taiwan Semiconductor Research Institute for the assistance with the research equipment.

\section{References}

1 Q. Ye, M. Chen, and W. Cai: Sol. Energy 184 (2019) 489. https://doi.org/10.1016/j.solener.2019.04.037

2 L. Wu, H. Zhang, F. Qin, X. Bai, Z. Ji, and D. Huang: Opt. Commun. 385 (2017) 205. https://doi.org/10.1016/j. optcom.2016.10.039

3 L. Scholtz, P. Šutta, P. Calta, P. Novák, M. Solanská, and J. Müllerová: Appl. Surf. Sci. 461 (2018) 249. https:// doi.org/10.1016/j.apsusc.2018.06.226

4 L. Yao and J. He: Prog. Mater Sci. 61 (2014) 94. https://doi.org/10.1016/j.pmatsci.2013.12.003

5 M. Farhat, T. C. Cheng, K. Q. Le, M. M. C. Cheng, H. Bağc1, and P. Y. Chen: Sci. Rep. 6 (2016) 19984. https:// doi.org/10.1038/srep19984

6 C. Y. Lin, K. Y. A. Lin, T. W. Yang, Y. C. Chen, and H. Yang: J. Colloid Interface Sci. 490 (2017) 174. https:// doi.org/10.1016/j.jcis.2016.11.064

7 T. Shao, F. Tang, L. Sun, X. Ye, J. He, L. Yang, and W. Zheng: Nanomaterials 9 (2019) 180. https://doi. org/10.3390/nano9020180

8 H. J. Jeong, Y. C. Kim, S. K. Lee, J. H. Yun, and J. H. Jang: Sol. Energy Mater. Sol. Cells 194 (2019) 177. https://doi.org/10.1016/j.solmat.2019.01.044

9 C. Atkinson, C. L. Sansom, H. J. Almond, and C. P. Shaw: Renewable Sustainable Energy Rev. 45 (2015) 113. https://doi.org/10.1016/j.rser.2015.01.015

10 B. Li, G. Niu, Y. Yi, X. W. Zhou, X. D. Liu, L. X. Sun, and C. Y. Wang: Superlattices Microstruct. 111 (2017) 57. https://doi.org/10.1016/j.spmi.2017.05.021

11 J. Y. Chen, W. L. Chang, C. K. Huang, and K. W. Sun: Opt. Express 19 (2011) 14411. https://doi.org/10.1364/ OE.19.014411

12 J. Taniguchi, E. Yamauchi, and Y. Nemoto: J. Phys. Conf. Ser. 106 (2008) 012011. http://dx.doi.org/10.1088/17426596/106/1/012011

13 C. H. Chang, J. A. Dominguez Caballero, H. J. Choi, and G. Barbastathis: Opt. Lett. 36 (2011) 2354. https://doi. org/10.1364/OL.36.002354

14 J. Wu, Z. X. Geng, Y. Y. Xie, Z. Y. Fan, Y. Su, C. Xu, and H. D. Chen: Nanomaterials 9 (2019). https://doi. org/10.3390/nano9010073

15 C. Phiphatanaphiphop, K. Leksakul, R. Phatthanakun, W. Busayaporn, C. Saiyasombat, P. Phothongkam, M. M. Rana, and H. Suzuki: J. Microelectromech. Syst. 29 (2020) 1264. https://doi.org/10.1109/ JMEMS.2020.3020130

16 Y. Yao, L. Zhang, T. Leydecker, and P. Samorì: J. Am. Chem. Soc. 140 (2018) 6984. https://doi.org/10.1021/ jacs.8b03526.

17 D. B. Dement, M. K. Quan, and V. E. Ferry: ACS Appl. Mater. Interfaces 11 (2019) 14970. https://doi. org/10.1021/acsami.9b01159

18 D. Kazazis, L. T. Tseng, and Y. Ekinci: Microelectron. Eng. 225 (2020) 111273. https://doi.org/10.1016/j. mee.2020.111273

19 S. K. Lam, A. Bendavid, and J. Du: Physica C 540 (2017) 38. https://doi.org/10.1016/j.physc.2017.07.009 
20 A. Kannegulla and L. J. Cheng: Nanotechnology 27 (2016) 36LT01. https://doi.org/10.1088/09574484/27/36/36LT01

21 Y. H. Kang, S. S. Oh, Y. S. Kim, and C. G. Choi: Microelectron. Eng. 87 (2010) 125. https://doi.org/10.1016/j. mee.2009.06.006

22 X. Wang, M. Sperling, M. Reifarth, and A. Böker: Small 16 (2020) 1906721. https://doi.org/10.1002/ smll.201906721

23 Z. Li, Y. Chen, X. Zhu, M. Zheng, F. Dong, P. Chen, L. Xu, W. Chu, and H. Duan: Nanotechnology 27 (2016) 365302. https://doi.org/10.1088/0957-4484/27/36/365302

24 Z. Li, C. Lu, A. B. Prakoso, M. Foldyna, R. Khoury, P. Bulkin, J. Wang, W. Chen, E. Johnson, and P. I. R. Cabarrocas: Nanomaterials 8 (2018) 626. https://doi.org/10.3390/nano8080626

25 L. Zaraska, G. D. Sulka, J. Szeremeta, and M. Jaskuła: Electrochim. Acta 55 (2010) 4377. https://doi. org/10.1016/j.electacta.2009.12.054

26 N. Wang, Y. Zhu, W. Wei, J. Chen, P. Li, and Y. Wen: Opt. Commun. 284 (2011) 4773. https://doi.org/10.1016/j. optcom.2011.05.063

27 J. Son, L. K. Verma, A. J. Danner, C. S. Bhatia, and H. Yang: Opt. Express 19 (2011) A35. https://doi. org/10.1364/OE.19.000A35

\section{About the Authors}

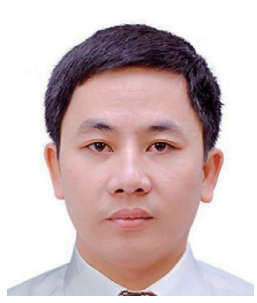

Trong-Linh Nguyen received his B.S. degree from Hung Yen University of Technology and Education, Vietnam, in 2008 and his M.S. degree from Hanoi University of Science and Technology, Vietnam, in 2012. He is currently pursuing his Ph.D. degree in the Department of Mechanical Engineering, National Kaohsiung University of Science and Technology, Taiwan. His research interests are in solar energy, desalination, and surface modification. (tronglinh.skh@gmail.com)

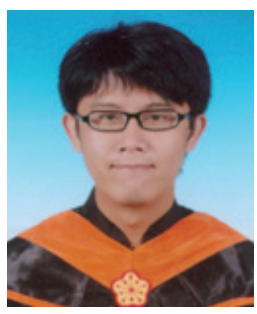

Chu-Chiang Chou received his B.S. and M.S. degrees from National Kaohsiung University of Science and Technology, Taiwan, in 2010 and 2012, respectively. His research interests are in semiconductor materials. (st2141@hotmail.com)

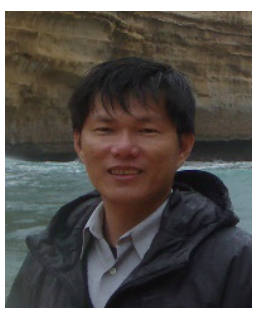

Te-Hua Fang received his B.S. degree from National Taiwan Institute of Technology, Taiwan, in 1992 and his M.S. and Ph.D. degrees from National Cheng Kung University, Taiwan, in 1994 and 2000, respectively. From 2001 to 2007, he was an assistant professor at Southern Taiwan University of Technology and National Formosa University, Taiwan. Since 2007, he has been a full professor at National Formosa University and National Kaohsiung University of Science and Technology. He is an IET Fellow. His research interests are in molecular dynamics, nanotechnology, materials, mechanics, and scanning probe microscopy. (fang.tehua@msa.hinet.net) 


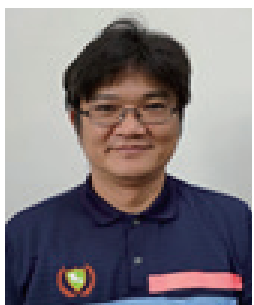

Tsung-Chieh Cheng received his Ph.D. degree from the Department of Mechanical Engineering, National Chiao Tung University in 2002. From 2002 to 2004, he worked at the Tainan Division of the National Nano Devices Laboratory (NDL) as an associate researcher. In 2005, he was promoted to the Material Characteristic Division in Hsinchu NDL. Since 2008, he has been a professor at the Department of Mechanical Engineering at National Kaohsiung University of Science and Technology, Taiwan, and was promoted to a full professor in 2016. His research interests are in surface modification, semiconductor materials, and solar energy. (tcchengme@nkust.edu.tw) 